\title{
Submillimeter and X-ray observations of an X class flare
}

\author{
C. G. Giménez de Castro ${ }^{1}$, G. Trottet ${ }^{2}$, A. Silva-Valio' ${ }^{1}$, S. Krucker ${ }^{3}$, J. E. R. Costa ${ }^{4}$, P. Kaufmann ${ }^{1,5}$, \\ E. Correia ${ }^{1,4}$, and H. Levato ${ }^{6}$
}

1 Centro de Rádio Astronomia e Astrofísica Mackenzie, R. da Consolação 896, 01302-907, São Paulo, SP, Brazil e-mail: guigue@craam.mackenzie.br, e-mail: guillermo.gimenez.de.castro@gmail.com

2 LESIA, Observatoire de Paris, Section de Meudon, 92195 Meudon, France

3 Space Sciences Laboratory, University of California, Berkeley, USA

4 Instituto Nacional de Pesquisas Espaciais, São José dos Campos, Brazil

5 Centro de Componentes Semicondutores, Universidade Estadual de Campinas, Campinas, Brazil

${ }^{6}$ Complejo Astronómico El Leoncito, CONICET, San Juan, Argentina

Received 10 March 2009 / Accepted 7 July 2009

\section{ABSTRACT}

\begin{abstract}
The GOES X1.5 class flare that occurred on August 30, 2002 at 1327:30 UT is one of the few events detected so far at submillimeter wavelengths. We present a detailed analysis of this flare combining radio observations from 1.5 to $212 \mathrm{GHz}$ (an upper limit of the flux is also provided at $405 \mathrm{GHz}$ ) and $\mathrm{X}$-ray. Although the observations of radio emission up to $212 \mathrm{GHz}$ indicates that relativistic electrons with energies of a few MeV were accelerated, no significant hard X-ray emission was detected by RHESSI above $250 \mathrm{keV}$. Images at 12-20 and 50-100 keV reveal a very compact, but resolved, source of about $\sim 10^{\prime \prime} \times 10^{\prime \prime}$. EUV TRACE images show a multi-kernel structure suggesting a complex (multipolar) magnetic topology. During the peak time the radio spectrum shows an extended flatness from $\sim 7$ to $35 \mathrm{GHz}$. Modeling the optically thin part of the radio spectrum as gyrosynchrotron emission we obtained the electron spectrum (spectral index $\delta$, instantaneous number of emitting electrons). It is shown that in order to keep the expected X-ray emission from the same emitting electrons below the RHESSI background at $250 \mathrm{keV}$, a magnetic field above $500 \mathrm{G}$ is necessary. On the other hand, the electron spectrum deduced from radio observations $\geq 50 \mathrm{GHz}$ is harder than that deduced from $\sim 70-250 \mathrm{keV} \mathrm{X-ray} \mathrm{data,}$ meaning that there must exist a breaking energy around a few hundred keV. During the decay of the impulsive phase, a hardening of the X-ray spectrum is observed which is interpreted as a hardening of the electron distribution spectrum produced by the diffusion due to Coulomb collisions of the trapped electrons in a medium with an electron density of $n_{\mathrm{e}} \sim 3-5 \times 10^{10} \mathrm{~cm}^{-3}$.
\end{abstract}

Key words. Sun: activity - Sun: flares - Sun: radio radiation - Sun: X-rays, gamma rays - Sun: particle emission

\section{Introduction}

During solar flares, a fraction of the released energy is used to accelerate electrons with energies well above $1 \mathrm{MeV}$. The interaction of these particles with the magnetic field of the flaring region produces gyrosynchrotron / synchrotron radiation observed at $\mathrm{cm}$ or smaller wavelengths (see e.g. Bastian et al. 1998; Pick \& Vilmer 2008, for reviews) and a bremsstrahlung continuum caused by Coulomb collisions observed with $\mathrm{X}$ - and $\gamma$-ray detectors. It was shown (e.g. Kundu et al. 1994) that the electron spectrum $N(E)$ determined by means of $\geq 30 \mathrm{GHz}$ radio observations is harder than that deduced from Hard X-ray (HXR) below a few hundred keV. However for a few events, the electron spectra were found consistent with spectra inferred from $\gamma$-ray continuum above $\sim 1 \mathrm{MeV}$ (Trottet et al. 1998, 2000). Since radio emission above $30 \mathrm{GHz}$ is produced mainly by electrons of a few MeV (see e.g. White \& Kundu 1992; Ramaty et al. 1994), these results have an impact on acceleration mechanism models, which are still, an open question in solar flare theory, and reinforces the need for good diagnostics of the $>1 \mathrm{MeV}$ particles.

Continuum $\mathrm{X}$ - and $\gamma$-ray detectors may observe photons from a few $\mathrm{keV}$ up to tens of $\mathrm{MeV}$, but have as a limitation the low sensitivity and / or high background in the high energy range. In the past three solar cycles only a few tens of flares have been observed above $1 \mathrm{MeV}$. On the other hand, radioastronomy at millimeter and submillimeter wavelengths is more efficient than the $\gamma$-ray detectors. Routine solar flare observations at 212 and $405 \mathrm{GHz}$ started in March 2001 with the Solar Submillimeter Telescope (SST, Kaufmann et al. 2001), installed in the Argentinean Andes. A few flares were also observed at 210,230 , and $345 \mathrm{GHz}$ with a receiver array installed at the focus of the Köln Observatory for Submillimeter and Millimeter Astronomy (KOSMA) telescope (Lüthi et al. 2004b,a). The first observations using such instruments showed that the spectrum above $100 \mathrm{GHz}$ is a continuation of the cm-wavelength optically thin spectrum (e.g. Trottet et al. 2002; Lüthi et al. 2004b) and extended the diagnostic tools of radio observations to higher energy (a few tens of $\mathrm{MeV}$ ) electrons. However, an unexpected upturn of the spectrum above $100 \mathrm{GHz}$ was reported for other $\geq$ M class events (e.g. Kaufmann et al. 2004; Lüthi et al. 2004a; Cristiani et al. 2008). The physical processes responsible for the production of the spectrum upturn are still unknown and are a subject of debate (Kaufmann \& Raulin 2006; Silva et al. 2007; Trottet et al. 2008).

In this paper, we present a combined analysis of the impulsive phase of the August 30, 2002, X class flare using RHESSI $\mathrm{X}$-ray observations and spatially unresolved radio data covering the range between 1.5 to $212 \mathrm{GHz}$ (and an upper limit for $405 \mathrm{GHz}$ ) obtained by different instruments. The event has been analyzed by different authors. Karlický et al. (2004) related radio observations between 0.8 and $2.0 \mathrm{GHz}$ and X-ray spectra and images from RHESSI. They found high-frequency drifting 
structures between 1327:38 and 1327:50 UT with a global drift of $-25 \mathrm{MHz} \mathrm{s}^{-1}$. The $10-20 \mathrm{keV} \mathrm{X}$-ray sources show a north-east displacement with a projected velocity of about $10 \mathrm{~km} \mathrm{~s}^{-1}$, while the $29-44 \mathrm{keV}$ emission is delayed by about 0.5 to $0.7 \mathrm{~s}$ after the radio drifting structure. Microwave observations of a short pulse during the onset of this event was analyzed by Giménez de Castro et al. (2006) who found a strikingly narrow spectrum that was explained as gyrosynchrotron emission of accelerated electrons with a maximum energy (high energy cutoff) of about $250 \mathrm{keV}$. Another event with similar properties was qualitatively discussed by Lüthi et al. (2004b). In this work we extend the analysis of Giménez de Castro et al. (2006) to the entire event. Moreover, we perform a quantitative analysis of the data which allows us to estimate the characteristics of the emitting electrons (energy spectrum, total number) and of the flaring region (density, magnetic field strength) that are necessary to account for the apparent discrepancy between X-ray and radio observations.

\section{Instrumentation}

The hard X-ray (HXR) and radio data used in the present analysis of the August 30, 2002 event were obtained with the NASA Reuven Ramaty High Energy Solar Spectroscopic Imager (RHESSI), the Solar Submillimeter Telescope (SST, installed in the Argentinean Andes), the nulling interferometer and patrol telescopes of the University of Bern (Switzerland), the Radio Solar Telescope Network (RSTN), and the Solar Radio-polarimeter of the Radio Observatory of Itapetinga (ROI, Brazil).

RHESSI provides imaging and spectral $\mathrm{HXR} / \gamma$-ray observations, with high spatial $(\sim 2$ arcsec) and spectral $(\sim 1 \mathrm{keV})$ resolution in the $\sim 3 \mathrm{keV}-17 \mathrm{MeV}$ energy range (Lin \& et al. 2002).

The SST (Kaufmann et al. 2000) operates simultaneously at 212 and $405 \mathrm{GHz}$ and with a time resolution of $1 \mathrm{~ms}$ for the present event. The focal system consists of four receivers at $212 \mathrm{GHz}$ and two at $405 \mathrm{GHz}$. At $212 \mathrm{GHz}$ this produces a cluster of beams that, in principle, allows us to determine the centroid of the emitting region whenever an event is detected (see Giménez de Castro et al. 1999, and references therein for details). During the August 30, 2002 flare, SST was tracking NOAA region 10095, with one of the two $405 \mathrm{GHz}$ beams pointing at the active region. At $212 \mathrm{GHz}$, the event was observed with only one beam so that it was not possible to estimate the centroid position of the emitting region. The antenna temperatures have been corrected for atmospheric attenuation (zenith optical depth $\tau_{212}=0.3$ nepers and $\tau_{405}=1.35$ nepers) and converted to flux density assuming that: (i) the source is much smaller than the beam size and; (ii) there is no important main-lobe gain correction due to a possible offset pointing. Since the HPBW of the beams are respectively $\sim 4^{\prime}$ and $2^{\prime}$ at 212 and $405 \mathrm{GHz}$, hypothesis (i) is justified here, because this event is very compact in the HXR domain (see Sect. 3.2). On the other hand, the projected position of the beam that observed the burst in the sky is separated by less than $30^{\prime \prime}$ from the HXR emitting region observed by RHESSI. As this is comparable to the absolute position uncertainty of the SST antenna, hypothesis; (ii) is also justified. It should be emphasized that a misalignment of $30^{\prime \prime}$ produces a main-lobe gain correction of less than $5 \%$ at $212 \mathrm{GHz}$ $\left(H P B W \sim 4^{\prime}\right)$.

The two-element nulling interferometer of the University of Bern provides total flux measurements at $89.4 \mathrm{GHz}$ with a sensitivity of $\sim 35$ s.f.u. ( 1 s.f.u. $=10^{-22} \mathrm{~W} \mathrm{~m}^{-2} \mathrm{~Hz}^{-1}$ ) and a time resolution of $31 \mathrm{~ms}$ (Lüthi et al. 2004b). Total flux densities at 11.8, 19.6, 35, and $50 \mathrm{GHz}$ were recorded by the patrol telescopes at

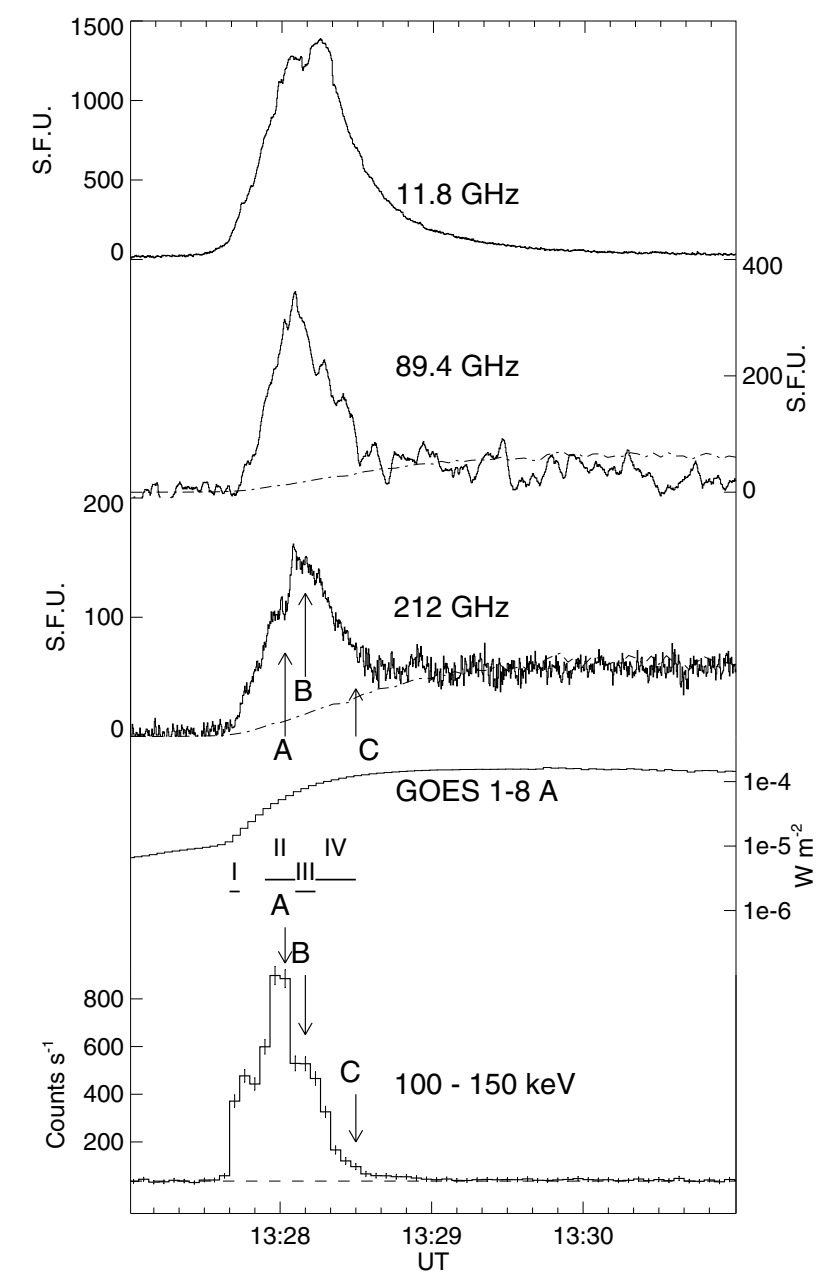

Fig. 1. Time evolution of the August 30, 2002 flare, at different radio frequencies and in selected SXR and HXR energy channels. At 89.4 and $212 \mathrm{GHz}$ the dashed curve represents the computed contribution of an isothermal source. A, B, and C indicate, respectively, characteristic time bins around the maximum of the HXR emission, the maximum of the $212 \mathrm{GHz}$ radiation, and the decay phase of the burst. Horizontal bars denote the time intervals I through IV (see text).

Bumishus (Switzerland) with a time resolution of $100 \mathrm{~ms}$. Total flux density measurements made at 1.415, 2.695, 4.995, 8.8, and $15.4 \mathrm{GHz}$ by the RSTN with a time resolution of $1 \mathrm{~s}$, and at $7 \mathrm{GHz}$ by the Solar Radio-polarimeter of the Radio Observatory of Itapetinga with $20 \mathrm{~ms}$ time resolution have also been used.

\section{Observations and data analysis}

The August 30, 2002 HXR and radio event starting at $\sim 1327: 30$ UT is associated with a GOES X1.5 SXR burst and, with a surprisingly small, $\mathrm{H} \alpha$ sub-flare which occurred in NOAA Active Region 10095 (N15 E74). This event was observed by RHESSI up to $\sim 250 \mathrm{keV}$ around its maximum and in a large part of the radio spectrum ranging from submillimeter to decameter and longer wavelengths. Figure 1 displays the time profile of the event in the 100-150 keV HXR band, in the SXR 1-8 channel; it also shows the total flux densities at 11.8, 89.4, and $212 \mathrm{GHz}$. The flare comprises an impulsive phase that starts at $\sim 1327: 40$ UT in the $100-150 \mathrm{keV}$ band and which lasts for about $60 \mathrm{~s}$. 


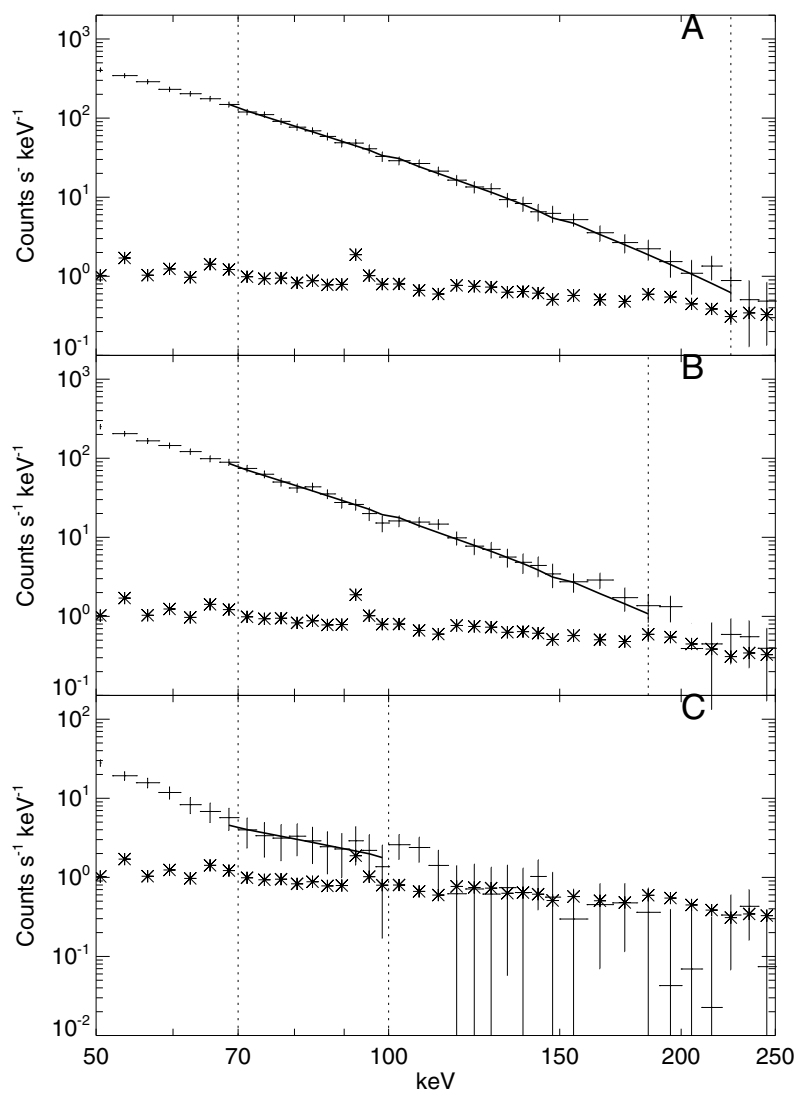

Fig. 2. Background subtracted count rate spectra measured by RHESSI (error bars) during time bins A, B, and C marked in Fig. 1. The asterisks show the background spectrum. The continuous lines show the best fit models (see text). The vertical dashed lines indicate the fitted energy range.

\subsection{Flux and spectra}

\subsubsection{Hard X-rays}

Spectral analysis of RHESSI data was performed between 1327:28 and 1328:36 UT. Count rate spectra were accumulated between 1327:38 and 1327:44 UT (before the thick shutter came in) and in all 4 second intervals between 1327:52 and 1328:32 UT using front detectors 1,3-6, 8 and 9. We applied pile up and decimation corrections. Each spectra consists of 77 energy bands between 3 and $250 \mathrm{keV}$. For each interval, spectral fitting was carried out for energies ranging from $40 \mathrm{keV}$ to the highest energy where count rates in excess of $2 \sigma$ above background are measured. It is found that the count rate spectra could be reasonably represented by considering either a single power law or a double power-law. In the latter case, the break energy lies around $70 \mathrm{keV}$. Since we are interested in the non-thermal $\mathrm{X}$-ray emission, we have restricted the analysis to the energies above $70 \mathrm{keV}$ using a single power-law of spectral index $\gamma$ for the trial photon spectra. Figure 2 displays examples of the fitted spectra for the time bins labeled A, B, and C in Fig. 1. The time evolution of $\gamma$ is shown in Fig. 3 .

\subsubsection{Radio}

At frequencies above $20 \mathrm{GHz}$ there is a time extended emission that lasts for tens of minutes after the impulsive phase (see Fig. 1 at 89.4 and $212 \mathrm{GHz}$ ). This gradual component is most likely of thermal origin because it seems to follow the SXR emission

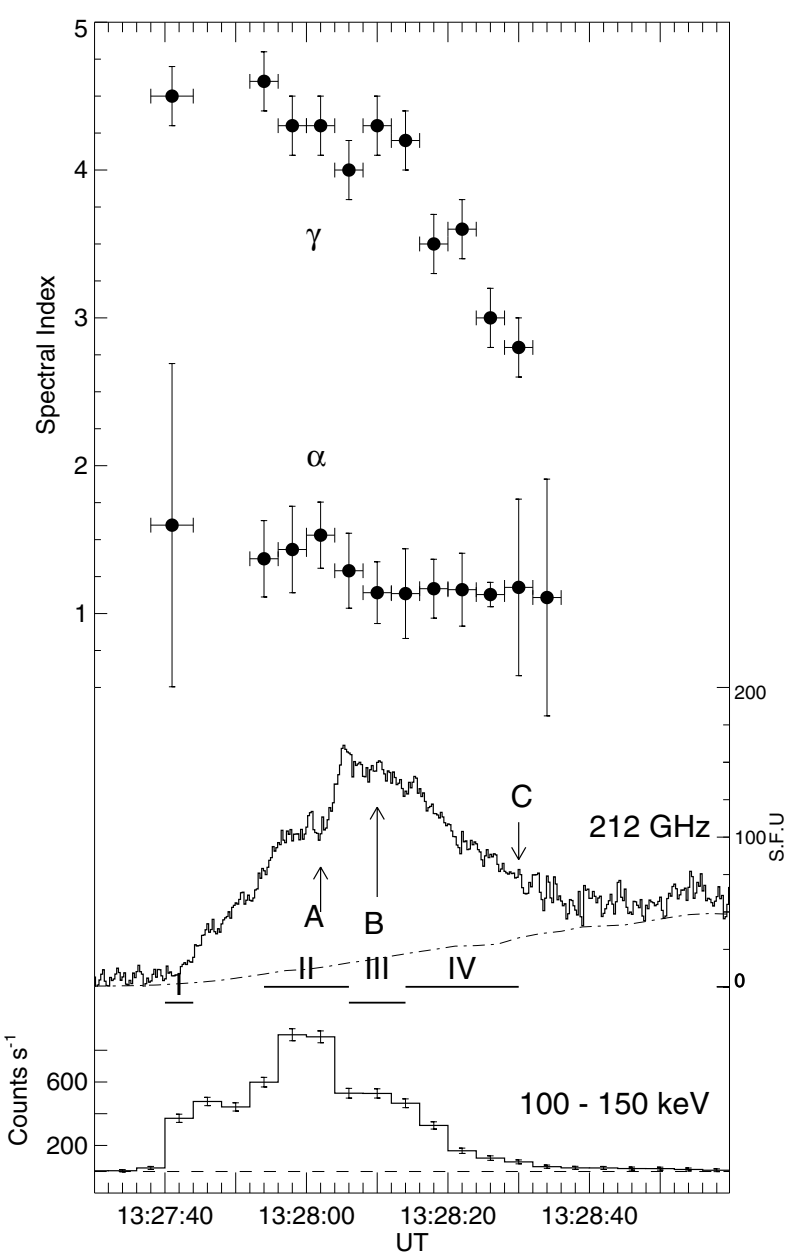

Fig. 3. From top to bottom: time evolution of the HXR spectral index $\gamma$ and of the radio spectral index $\alpha$ (dots), of the radio flux density at $212 \mathrm{GHz}$ (the dot-dashed curve represents the computed contribution of an isothermal source), and of the $100-150 \mathrm{keV}$ emission. Horizontal bars show the time intervals I through IV (see text).

from GOES and has no HXR counterpart. The comparison of the $>20 \mathrm{GHz}$ time evolution with that of the 1-8 $\mathrm{A}$ SXR indicates that this thermal component may have started at the beginning of the impulsive phase and therefore should be subtracted from the total flux densities in order to estimate the non thermal emission of the radio burst. For this we consider that the thermal emission arises from the hot thermal source that produces the SXR emission observed by GOES. We computed the free-free flux density of an isothermal source with temperature and emission measure (EM) derived from GOES 8 observations. A source size of $60^{\prime \prime}$ provides a reasonable agreement with the observations as illustrated in Fig. 1 (dot-dash lines at 89.4 and $212 \mathrm{GHz}$ curves). Figure 4 shows the non-thermal radio spectrum for the three time bins marked A, B and C in Fig. 1 that correspond, respectively, to the maximum of the $100-150 \mathrm{keV}$ emission, to the maximum of the $212 \mathrm{GHz}$, and to the decay of the impulsive phase. The main characteristics of the radio spectra can be summarized as follows:

from 1 to $7 \mathrm{GHz}$ : the spectrum increases with frequency and can be represented by a power law with spectral index $\sim 1$;

from 7 to $35 \mathrm{GHz}$ : there is a plateau observed during the impulsive phase (Fig. 4A, B). During the decay of the impulsive phase the spectrum gradually evolves exhibiting a rather well defined turnover frequency around $5 \mathrm{GHz}$ (Fig. 4C); 


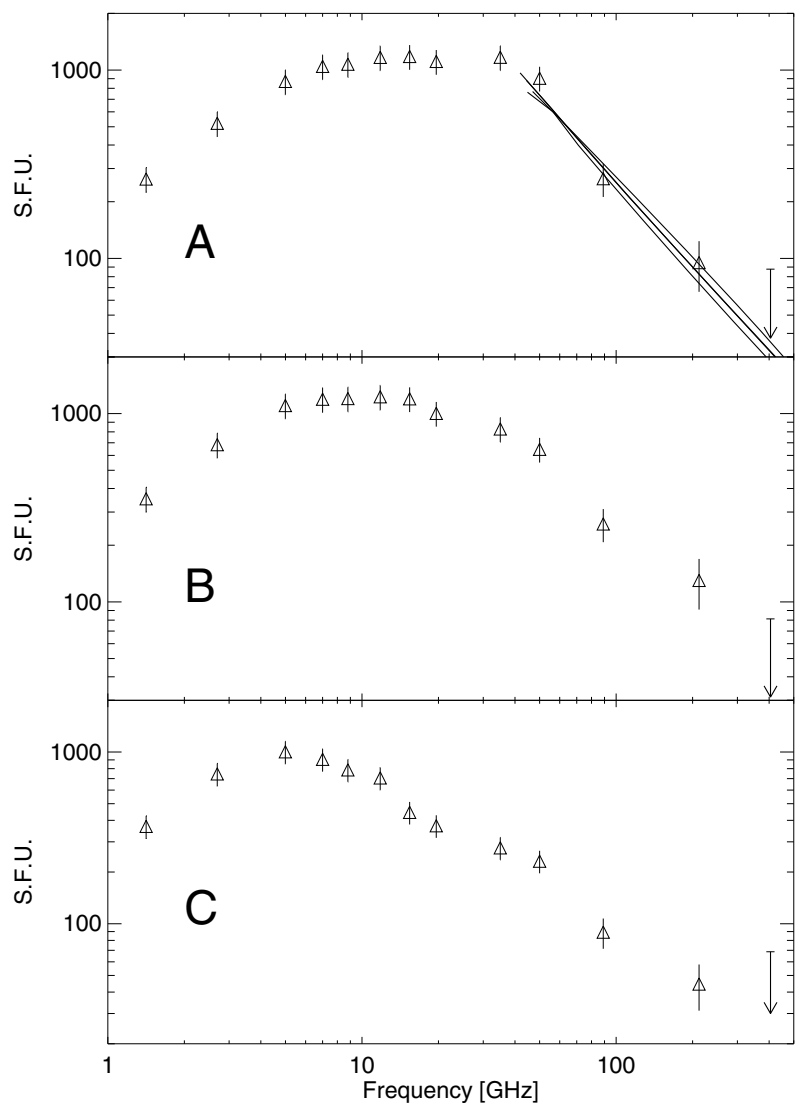

Fig. 4. The radio spectrum at instants labeled A, B, and C. (see Fig. 1) Solid curves represent the homogeneous gyrosynchrotron solution fits discussed in Sect. 4.3.

above $50 \mathrm{GHz}$ : the flux density is roughly proportional to $v^{-\alpha}$.

Figure 3 shows the time evolution of $\alpha$.

\subsection{HXR images}

Figure 5 displays RHESSI contours in the $12-20 \mathrm{keV}$ and 50-100 keV bands overlaid on a $195 \AA$ TRACE image taken at 1327:31 UT for the four intervals marked by horizontal bars in Fig. 1. Intervals I and II cover the first two 100-150 keV peaks, interval III spreads over the maximum of the $212 \mathrm{GHz}$, while interval IV extends over the HXR decay phase. RHESSI images were obtained by applying the PIXON algorithm (Metcalf et al. 1996) and by using front detector segments 1 to 6 . Detector 2 was not used for the $12-20 \mathrm{keV}$ images. The TRACE image was taken close to the onset of the radio and HXR impulsive peaks. The figure shows that the impulsive phase of the flare was triggered within a complex pattern of bright EUV features in a compact region $\left(10^{\prime \prime} \times 10^{\prime \prime}\right)$. Unfortunately, subsequent $195 \AA$ TRACE images are saturated until 1350 UT. During intervals I and II, the 50-100 keV emission (solid contours) arises from two compact regions $\left(\sim 3-7^{\prime \prime}\right)$ overlaying bright EUV structures. During the rest of the event (intervals III to IV), only the southeast source is observed at 50-100 keV. During the whole event the lower energy HXR (12-20 keV, dashed contours) arise predominantly from a single source close to the southeast of the $50-100 \mathrm{keV}$ emitting region. However, the interpretation of the HXR images at $50 \mathrm{keV}$ for interval III and IV is inconclusive as up to half of the counts are due to pile up of thermal photons. To correct pile up in images is very difficult as the corrections

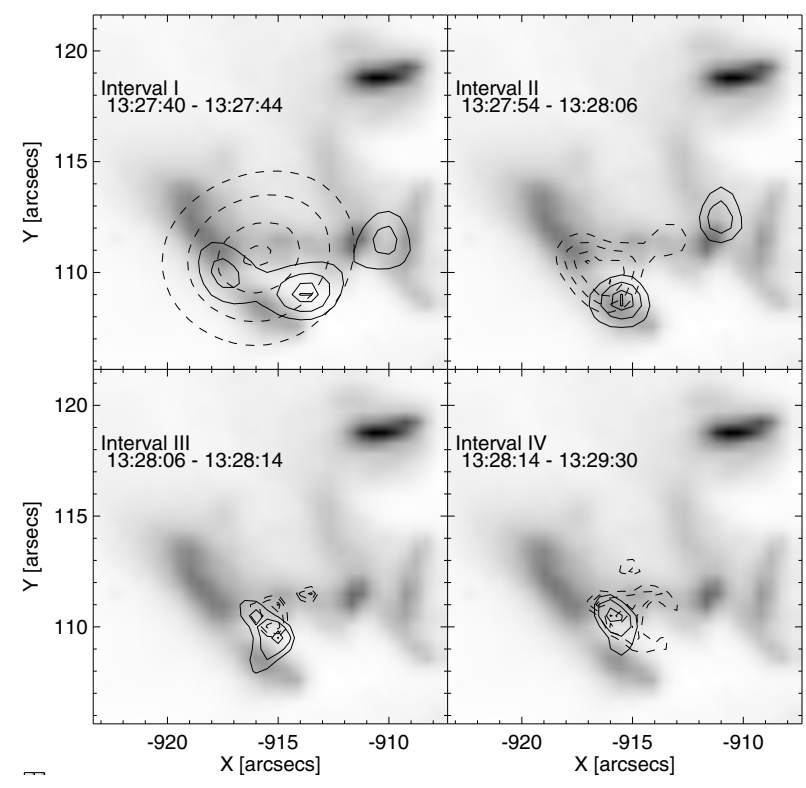

Fig. 5. RHESSI emitting regions of 50-100 keV (solid contours) and of 12-20 keV (dashed contours) for different time intervals superimposed on a negative $195 \AA$ image taken by TRACE at 1327:31 UT. Contour levels are: 50, 70, 90, and 99\% of the image maximum.

should be done in the modulated light curves. Currently, the RHESSI software does not provide pile up correction in images.

\section{Discussion}

EUV observations close to the onset of the impulsive phase of the August 30, 2002 event reveal that this flare arose from a compact region with a multi kernel structure that suggests a complex (multipolar) magnetic field topology. The HXR emitting sources are observed in association with different EUV bright structures, suggesting that they are located at the footpoints of different magnetic loops (see Fig. 5). The compactness of this flare is further supported by the fact that such an X class event produced only an $\mathrm{H} \alpha$ sub-flare. In the following, we discuss some peculiarities of this HXR and radio event:

- the unusually flat radio spectrum between 7 and $35 \mathrm{GHz}$ during most of the impulsive phase (intervals I to III on Fig. 1);

- the lack of significant $\gamma$ ray emission detected by RHESSI in the $\mathrm{MeV}$ domain, although $\geq 90 \mathrm{GHz}$ radio data indicate that relativistic electrons were produced during the flare;

- the strong hardening of the $>70 \mathrm{keV}$ HXR spectrum during the decay of the impulsive phase.

Figure 3 (top) presents the time evolution of the HXR spectral index $\gamma$ obtained assuming that the photon distribution is a single power law from $70 \mathrm{keV}$ to the highest energy where count rates are above the background (see Sect. 3.1.1). During intervals I to III, the spectral index does not vary significantly, remaining around $\gamma=4.3 \pm 0.3$, however during interval IV there is a spectral hardening. We consider that intervals I, II, and III correspond to three successive different particle injections:

- during intervals I and II, the 100-150 keV emission is consistent with thick-target emission because it arises predominantly from footpoint sources. Furthermore there is no long-term coronal trapping because the HXR peaks occur simultaneously at all energies within the RHESSI time resolution (4 s). Thus, the time evolution of the HXR emission 
mimics that of the electron injection into the thick-target region;

- taking into account the above arguments, interval III corresponds to a third injection which is reflected by the shoulder in the 100-150 keV time profile range and corresponds to the maximum of the $212 \mathrm{GHz}$ emission;

- the hardening of the HXR spectrum during interval IV may be indicative of some trapping.

\subsection{The flat radio spectrum}

It is well documented that cm-mm emission comes from gyrosynchrotron radiation of energetic relativistic electrons propagating in the magnetic structures (e.g. Pick \& Vilmer 2008, and references therein). The radio spectra of the present event are indeed reminiscent of this emission process. In a uniform magnetic field, the emission would lead to a spectral index larger than 2.5 in the optically thick region and a rather well defined turnover frequency. However, the spectral index for the frequency range 1-7 GHz (optically thick emission) remains around or below 1 during the whole event. And until the decay of the impulsive phase (interval IV) the radio spectrum is flat between 7 and $35 \mathrm{GHz}$ (see Fig. 4). Both these characteristics are indications that radio emitting electrons propagate in a highly inhomogeneous magnetic field (e.g. Dulk 1985; Klein et al. 1986; Lee et al. 1994). This inhomogeneous magnetic field interpretation is consistent with the complex magnetic structure revealed by TRACE and RHESSI images. During the decay of the impulsive phase, a well defined turnover frequency gradually becomes better defined. At the same time, the X-ray emission arises from a single source, so that the observed radio spectrum is closer to that expected from a homogeneous source.

Ramaty \& Petrosian (1972) explained flat microwave spectra as observed by Hachenberg \& Wallis (1961) by including the free-free absorption of a cold medium uniformly mixed in the homogeneous gyrosynchrotron source region. Indeed: (i) at low frequencies, where both gyrosynchrotron and free-free opacities are $>1$, the radio flux increases with frequency; (ii) at frequencies for which the gyrosynchrotron opacity is $<1$ while the freefree opacity remains $>1$, a plateau is observed because while the gyrosynchrotron emission starts to decrease the free-free emission is still increasing; (iii) at higher frequencies both emissions are optically thin and the radio spectrum decreases with frequency. The observation of simultaneous brightenings and line broadening of hot $\left(\sim 10^{7} \mathrm{~K}\right)$ and cold $\left(\sim 10^{4} \mathrm{~K}\right)$ plasmas during a solar limb flare (Kliem et al. 2002) provides some support to the Ramaty \& Petrosian hypothesis. In addition to magnetic field inhomogeneities, free-free absorption may also contribute to provide the observed flat radio spectrum. In that case, as the flare evolves, the free-free opacity should decrease in order to allow lower frequency radiation to become optically thin. Since freefree opacity is proportional to $n_{\mathrm{p}}^{2} T_{\mathrm{p}}^{-3 / 2}\left(n_{\mathrm{p}}, T_{\mathrm{p}}\right.$ medium density and temperature, respectively, and assuming $\left.n_{\mathrm{e}}=n_{\mathrm{p}}\right)$, this would imply either a decrease of the density, or an increase of the temperature, or an increase of both. In the latter case the temperature should increase faster than the density.

Above $50 \mathrm{GHz}$ (optically thin emission), the radiation, which is mostly emitted by highly relativistic electrons, is not affected by the medium, and the spectral radio index $\alpha$ is only related to the index of the instantaneous electron distribution $\delta$. For the present event $\alpha$ remains between 1.1 and 1.3 (Fig. 3). Considering the ultra-relativistic case as a gross approximation, this leads to $\delta=2 \alpha+1=3.2-3.6$ (Dulk 1985).

\subsection{HXR spectral hardening during the decay of the impulsive phase}

During interval IV the HXR spectral index $\gamma$ decreases from 4.1 to 2.8 (Fig. 3). This provides some indication that a significant amount of HXR was produced by trapped electrons, the diffusion rate being governed by Coulomb collisions. For the weak-diffusion limit case, the trapping time $t^{\text {trap }} \simeq t^{\text {def }}$, with $t^{\text {def }}$ the characteristic deflection time, which is given by Trubnikov (1965) and Melrose \& Brown (1976)

$t^{\mathrm{def}} \simeq \frac{E}{2}\left(\frac{\mathrm{d} E}{\mathrm{~d} t}\right)_{\text {coll }}^{-1}$,

where $(\mathrm{d} E / \mathrm{d} t)_{\text {coll }}$, the electron energy loss rate due to Coulomb collisions, is approximated by (Bai \& Ramaty 1979)

$\left(\frac{\mathrm{d} E}{\mathrm{~d} t}\right)_{\text {coll }}= \begin{cases}-4.910^{-9} E^{-0.5} n_{\mathrm{e}} & E \leq 160 \mathrm{keV} \\ -3.810^{-10} n_{\mathrm{e}} & E>160 \mathrm{keV}\end{cases}$

with $E$ the electron energy in $\mathrm{keV}$ and $n_{\mathrm{e}}$ the medium electron density in $\mathrm{cm}^{-3}$.

We consider that the injection of electrons in the HXR emitting region stopped at 1328:14 UT because it is the last time bin where $\gamma$ remains almost constant (see Fig. 3). Under these conditions the time evolution of the distribution of the trapped electrons is approximately given by (Aschwanden 1998)

$N(E, t) \propto E^{-\delta} \exp \left(-\frac{t}{t^{\mathrm{def}}}\right)$,

with $\delta$ the electron index at the end of the injection. The photon flux at energy $\epsilon$ as a function of time $t$ may be written as (see e.g. Brown 1971)

$F(\epsilon, t)=n_{\mathrm{e}} \int_{\epsilon}^{\infty} \sigma(\epsilon, E) v(E) N(E, t) \mathrm{d} E$,

where $v(E)$ is the electron velocity corresponding to energy $E, \sigma(\epsilon, E)$ is the bremsstrahlung differential cross-section per unit photon energy $\epsilon$ for an electron of energy $E$. For $\sigma(E)$ we adopted the Bethe-Heitler electron - proton bremsstrahlung cross-section. It should be noted that the photon flux produced by precipitated electrons has a time evolution as $F(\epsilon, t)$ (Melrose $\&$ Brown 1976). Therefore, for a given $\delta$, the time evolution of the photon flux depends only on $n_{\mathrm{e}}$. By using Eq. (4) we computed the expected photon flux $F(\epsilon, t)$ and compared its time evolution at different energies $\epsilon$ with that observed by RHESSI during the decaying of the impulsive phase. Comparing the expected and observed time profiles at 70, 100, and $150 \mathrm{keV}$, we obtained reasonable agreement for $n_{\mathrm{e}} \sim 3-5 \times 10^{10} \mathrm{~cm}^{-3}$ when $\delta$ ranges between 3.5 and 5 , which correspond respectively to thin and thick target limits for $\gamma \sim 4$. Krucker et al. (2008) find similar electron densities for coronal $\gamma$-ray emission during flares.

Spectral hardening has been reported during the impulsive phase of long duration GOES X class flares and associated with non thermal footpoint bremsstrahlung (Qiu et al. 2004; Saldanha et al. 2008). Grigis \& Benz (2008) analyzed the spectral hardening during the gradual phase of great flares and concluded that the cause is the continuing acceleration with longer trapping in the accelerator before escape. Kiplinger (1995) has shown that the hardening is associated with SEP events.

\subsection{Relationship between $H X R$ and radio emission}

The absence of HXR emission $>250 \mathrm{keV}$ while we observe radio emission above $50 \mathrm{GHz}$ can be used to constrain the high 
Table 1. Derived characteristic of radio radiating electrons for different magnetic field strengths and view angle equal to $84^{\circ}$.

\begin{tabular}{ccc}
\hline \hline $\begin{array}{c}\boldsymbol{B} \\
\text { (Gauss) }\end{array}$ & $N(>25 \mathrm{keV}) \times 10^{33}$ & $\begin{array}{c}K \times 10^{29} \\
\left(\mathrm{e}^{-} \mathrm{MeV}^{-1}\right)\end{array}$ \\
\hline 500 & 38.1 & 76.0 \\
750 & 15.0 & 30.0 \\
1000 & 7.5 & 15.0 \\
1600 & 1.5 & 3.0 \\
\hline
\end{tabular}

energy electron distribution, the magnetic field, and the trapping time in the radio emitting region. For that, we consider that non thermal electrons are injected in coronal loops. The radio emission is produced in the coronal portion of these loops where they become partially trapped while precipitating electrons produce the HXR radiation by thick target interaction at the loop footpoints. Since no spatially resolved radio data are available for this event and the optically thin part of the radio spectrum does not depend on the structure details of the medium, we used a homogeneous model to derive the mean parameters of the radio source and emitting electrons. For that, we computed the radio optically thin emission by using the numerical code for a gyrosynchrotron source with a homogeneous ambient density and magnetic field and an isotropic electron distribution developed by Ramaty (1969) and corrected by Ramaty et al. (1994) ${ }^{1}$. The instantaneous electron distribution in the radio source was taken as $N(E)=K E^{-\delta}$, where $K$ is the number of electrons per $\mathrm{MeV}$ at $1 \mathrm{MeV}$ and the energy $E$ is in $\mathrm{MeV}$. The angle between the observer and the magnetic field (view-angle) was set to $84^{\circ}$ (the maximum allowed value in Ramaty's solution) in order to obtain the lower limit of the total number of electrons necessary to produce a given radio spectrum for a given magnetic field strength. For a view-angle of $45^{\circ}$, as is usually assumed, the computed number of electrons is roughly twice that obtained for $84^{\circ}$. Table 1 displays the values of the instantaneous total number of electrons above $25 \mathrm{keV} \mathrm{N}(>25 \mathrm{keV})$ and $K$ obtained for different values of the magnetic field at the maximum of the $100-150 \mathrm{keV}$ HXR (time bin A) . $N(>25 \mathrm{keV}$ ) was computed for $\delta=3.4$ which provides the best fit to the radio data. This is in agreement with the value of $\delta$ inferred from the slope of the optically thin part of the radio spectrum $\alpha$ (see Sect. 4.1).

The mean electron flux $\dot{N}(E)$ entering the thick target HXR source is $\dot{N}(E) \simeq N(E) / t^{\text {trap }}$, where $t^{\text {trap }}$ is the time spent by the electrons in the radio source. The thick target photon emission from these precipitating electrons was calculated by using a numerical code that takes into account both electron-proton and electron-electron collisions. The photon spectrum is then convolved with the RHESSI response matrix to get the corresponding count rate spectrum. Figure 1 shows that within the RHESSI time resolution (4 s), the $89.4 \mathrm{GHz}, 212 \mathrm{GHz}$ and $100-150 \mathrm{keV}$ count rates show simultaneous peaks during intervals I to III. The electron trapping time $t^{\text {trap }}$ can thus be considered independent of energy as a first approximation. This suggest that during intervals I to III the precipitation rate is more likely governed by wave-particle interactions (turbulent trapping) than by Coulomb collisions (e.g. Vilmer 1987). The computations were then carried out for $10^{-2} \leq t^{\text {trap }} \leq 4 \mathrm{~s}$, the lower and upper limits correspond respectively to free streaming of the electrons in a compact loop $\left(\sim 10^{\prime \prime}\right)$, and to the RHESSI time resolution. Figure 6 displays the RHESSI expected count rates in the 250$265 \mathrm{keV}$ band as a function of $t^{\text {trap }}$ for different values of the

\footnotetext{
1 See http://1heawww.gsfc.nasa.gov/users/ramaty
}

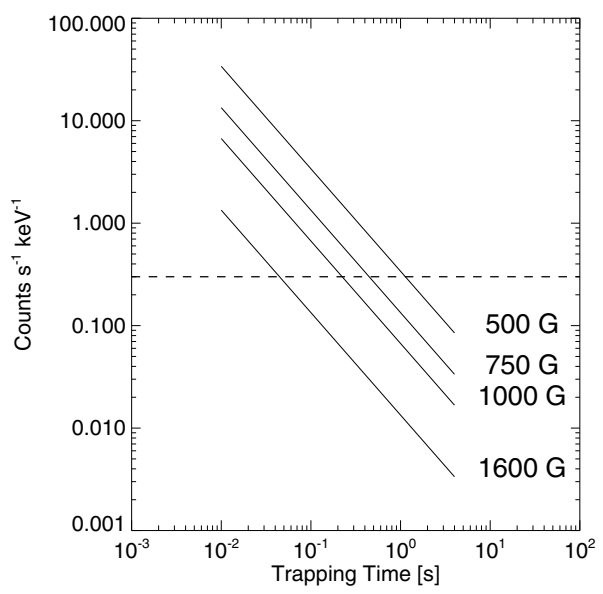

Fig. 6. Expected RHESSI count rates at $250 \mathrm{keV}$ as a function of the emitting electron trapping time for different magnetic field strengths and view angle equal to $84^{\circ}$. Each solid line represents a different solution determined by a different magnetic field. The dashed horizontal line represents the measured background count rate at $250 \mathrm{keV}$.

magnetic field. The dashed horizontal line corresponds to the RHESSI count rate in this channel, the highest energy where this event was detected. We conclude that the mean magnetic field strength should be greater than about $500 \mathrm{G}$ to keep the thick target photon flux expected from radio emitting electrons with trapping times smaller than $1 \mathrm{~s}$ below the detection limit of RHESSI

It has been shown that the thick target spectral index $\gamma$ is bounded between $\delta-1.5 \leq \gamma \leq \delta-1$, the lower limit corresponding to turbulent trapping of electrons with energies above a few $100 \mathrm{keV}$, whereas the higher limit is set by free propagating electrons with energies below a few $100 \mathrm{keV}$ (see e.g. Trottet et al. 1998, and references therein.). Therefore if $\delta=3.4$, the HXR emission of these electrons should have an index between $1.9 \leq \gamma \leq 2.4$ which is significantly smaller than the observed values, $\sim 4.3$, during intervals I to III (see Fig. 3). This is in accordance with previous works that show that radio high frequency emission is generated by electrons with energies above $\sim 500 \mathrm{keV}$ with an electron index harder than the $<500 \mathrm{keV}$ electrons (White \& Kundu 1992; Kundu et al. 1994; Trottet et al. 1998; Silva et al. 2000).

\section{Summary}

In this paper we have analyzed X-ray observations from RHESSI and radio data obtained at submillimeter wavelengths by the Solar Submillimeter Telescope (SST) of the X1.5 event that occurred in Active Region 10095 on August 30, 2002, at 1327:30 UT, complemented with radio observations from 1.5 to $89.4 \mathrm{GHz}$ from other instruments. EUV images from TRACE provided information about the source emitting region.

The radio spectrum above $100 \mathrm{GHz}$ is the continuation of the optically thin microwave spectrum, therefore does not belong to the so called $\mathrm{THz}$ bursts (Kaufmann et al. 2004), although it is an X Class flare. We summarize below our main findings:

- The magnetic structure of the flare is complex and highly inhomogeneous. This is revealed by the $50-100 \mathrm{keV}$ and EUV images. Such an inhomogeneous source may produce the flatness in the radio spectrum observed between 7 and $35 \mathrm{GHz}$, although we do not discard the free-free absorption. 
- The electron spectrum $N(E)$ above $1 \mathrm{MeV}$ is harder than that at energies below a few hundred $\mathrm{keV}$.

- Modeling simultaneously the expected RHESSI count rate and the expected gyrosynchrotron emission, we obtain $500 \mathrm{G}$ as a lower limit for the mean magnetic field of the flaring region.

- The time evolution of the spectral index deduced from X-ray observations at the end of interval III suggests that trapped electrons are diffused by Coulomb collisions. This leads to a mean ambient electron density of $3-5 \times 10^{10} \mathrm{~cm}^{-3}$, typical of the low corona / upper chromosphere and is compatible with previous results (Krucker et al. 2008) and with the small size of the EUV pattern observed by TRACE, which also suggests that the flaring region does not extend high in the corona.

Finally it should be emphasized that radio observation in the sub-THz domain provide a unique tool to constrain acceleration model because they constitute a more sensitive diagnostic of ultra-relativistic electrons than present $\mathrm{HXR} / \gamma$-ray measurements.

Acknowledgements. This research was partially supported by Brazil Agencies FAPESP, CNPq and Mackpesquisa, and Argentina Agency CONICET. CGGC also thanks the Observatory of Paris in Meudon, that supported his stay to finish the present work. The authors are in debt to A. Magun and T. Lüthi who provided the calibrated data of the Bern patrol telescopes and of the nulling interferometer at $89.4 \mathrm{GHz}$. They would also like to thank Dr. Lidia van Driel-Gesztelyi for helpful discussions.

\section{References}

Aschwanden, M. J. 1998, ApJ, 502, 455

Bai, T., \& Ramaty, R. 1979, ApJ, 227, 1072

Bastian, T. S., Benz, A. O., \& Gary, D. E. 1998, ARA\&A, 36, 131

Brown, J. C. 1971, Sol. Phys., 18, 489

Cristiani, G., Giménez de Castro, C. G., Mandrini, C. H., et al. 2008, A\&A, 492, 215
Dulk, G. A. 1985, ARA\&A, 23, 169

Giménez de Castro, C. G., Raulin, J. P., Makhmutov, V. S., Kaufmann, P., \& Costa, J. E. R. 1999, A\&AS, 140, 373

Giménez de Castro, C. G., Costa, J. E. R., Silva, A. V. R., et al. 2006, A\&A, 457, 693

Grigis, P. C., \& Benz, A. O. 2008, ApJ, 683, 1180

Hachenberg, O., \& Wallis, G. 1961, Z. Astrophys., 52, 42

Karlický, M., Fárník, F., \& Krucker, S. 2004, A\&A, 419, 365

Kaufmann, P., \& Raulin, J.-P. 2006, Phys. Plasmas, 13, 701

Kaufmann, P., Costa, J. E. R., Correia, E., et al. 2000, in High Energy Solar Physics Workshop - Anticipating Hessi, ed. R. Ramaty, \& N. Mandzhavidze, 318, ASP Conf. Ser., 206

Kaufmann, P., Costa, J. E. R., Giménez de Castro, C. G., et al. 2001, in Proc. of the SBMO/IEEE MTT-S International Microwave and Optoelectronics Conference, 439

Kaufmann, P., Raulin, J. P., de Castro, C. G. G., et al. 2004, ApJ, 603, L121

Kiplinger, A. L. 1995, ApJ, 453, 973

Klein, K.-L., Trottet, G., \& Magun, A. 1986, Sol. Phys., 104, 243

Kliem, B., Dammasch, I. E., Curdt, W., et al. 2002, ApJ, 568, L61

Krucker, S., Hurford, G. J., MacKinnon, A. L., Shih, A. Y., \& Lin, R. P. 2008, ApJ, 678, L63

Kundu, M. R., White, S. M., Gopalswamy, N., et al. 1994, ApJS, 90, 599

Lüthi, T., Lüdi, A., \& Magun, A. 2004a, A\&A, 420, 361

Lüthi, T., Magun, A., \& Miller, M. 2004b, A\&A, 415, 1123

Lee, J. W., Gary, D. E., \& Zirin, H. 1994, Sol. Phys., 152, 409

Lin, R. P., Dennis, B. R., Hurford, G. J., et al. 2002, Sol. Phys., 210, 3

Melrose, D. B., \& Brown, J. C. 1976, MNRAS, 176, 15

Metcalf, T. R., Hudson, H. S., Kosugi, T., Puetter, R. C., \& Pina, R. K. 1996, ApJ, 466, 585

Pick, M., \& Vilmer, N. 2008, A\&A Rev., 6

Qiu, J., Lee, J., \& Gary, D. E. 2004, ApJ, 603, 335

Ramaty, R. 1969, ApJ, 158, 753

Ramaty, R., \& Petrosian, V. 1972, ApJ, 178, 241

Ramaty, R., Schwartz, R. A., Enome, S., et al. 1994, ApJ, 436, 941

Saldanha, R., Krucker, S., \& Lin, R. P. 2008, ApJ, 673, 1169

Silva, A. V. R., Wang, H., \& Gary, D. E. 2000, ApJ, 545, 1116

Silva, A. V. R., Share, G. H., Murphy, R. J., et al. 2007, Sol. Phys., 245, 311

Trottet, G., Vilmer, N., Barat, C., et al. 1998, A\&A, 334, 1099

Trottet, G., Rolli, E., Magun, A., et al. 2000, A\&A, 356, 1067

Trottet, G., Raulin, J. P., Kaufmann, P., et al. 2002, A\&A, 381, 694

Trottet, G., Krucker, S., Lüthi, T., et al. 2008, ApJ, 678, 509

Trubnikov, B. A. 1965, Rev. Plasma Phys., 1, 105

Vilmer, N. 1987, Sol. Phys., 111, 207

White, S. M., \& Kundu, M. R. 1992, Sol. Phys., 141, 347 